\title{
The price of selfish behavior in bilateral network formation
}

\section{Citation}

Corbo, Jacomo and David Parkes. 2005. The price of selfish behavior in bilateral network formation. In Proceedings of the twenty-fourth annual ACM symposium on Principles of distributed computing (PODC 2005), Las Vegas, NV, July 17-20, 2005: 99-107.

\section{Published Version}

10.1145/1073814.1073833

\section{Permanent link}

http://nrs.harvard.edu/urn-3:HUL.InstRepos:32713662

\section{Terms of Use}

This article was downloaded from Harvard University's DASH repository, and is made available under the terms and conditions applicable to Other Posted Material, as set forth at http:// nrs.harvard.edu/urn-3:HUL.InstRepos:dash.current.terms-of-use\#LAA

\section{Share Your Story}

The Harvard community has made this article openly available.

Please share how this access benefits you. Submit a story.

Accessibility 


\title{
The Price of Selfish Behavior in Bilateral Network Formation*
}

\author{
Jacomo Corbo, David C. Parkes \\ Division of Engineering and Applied Sciences \\ 33 Oxford Street, Maxwell Dworkin, Harvard University \\ \{jacomo, parkes\}@eecs.harvard.edu
}

\begin{abstract}
Given a collection of selfish agents who wish to establish links to route traffic among themselves, the set of equilibrium network topologies may appear quite different from the centrally enforced optimum. We study the quality (price of anarchy) of equilibrium networks in a game where links require the consent of both participants and are negotiated bilaterally, and compare these networks to those generated by an earlier model due to Fabrikant et al. [10] in which links are formed unilaterally. We provide a partial characterization of stable and efficient networks in the bilateral network formation game, and provide examples of stable networks that are not Nash graphs in the unilateral game. We develop an upper and lower bound on the price of anarchy of the bilateral game. An empirical analysis demonstrates that the average price of anarchy is better in the bilateral connection game than the unilateral game for small link costs but worse as links become more expensive. In the process, a relationship between link-based graph stability and two game-theoretic equilibrium notions is discussed. This relationship helps to develop a partial geometric characterization of equilibrium graphs in the bilateral connection game.
\end{abstract}

\begin{abstract}
*This is a revised version of the published PODC'05 paper, fixing a number of errors. The discussion is modified in various places to reflect these corrections. The lower and upper bounds on price of anarchy (PoA) in the bilateral connection game (BCG) are unaffected (Propositions 3 and 4). The proof of Proposition 5 that all Nash graphs of the unilateral connection game (UCG) are pairwise stable in the BCG (for the same link cost) was incorrect, and the result is restated here for trees. Since this paper was published, Corollary 2 that the PoA is no better in the BCG than the UCG, whatever the link cost, has been established through results of Demaine et al. [8], who also established that our upper-bound for the BCG is tight. Proposition 2 that pairwise-stable networks in BCG are achievable as a proper equilibrium was incorrect and is restated here for a special case (thanks to Éva Tardos, who pointed out a problem with Lemma 2.) Definitions 2 and 3 are also slightly modified to replace a weak inequality with a strict inequality when adding an edge (thanks to Lasse Kliemann).
\end{abstract}

Permission to make digital or hard copies of all or part of this work for personal or classroom use is granted without fee provided that copies are not made or distributed for profit or commercial advantage and that copies bear this notice and the full citation on the first page. To copy otherwise, to republish, to post on servers or to redistribute to lists, requires prior specific permission and/or a fee.

PODC'05, July 17-20, 2005, Las Vegas, Nevada, USA.

Copyright 2005 ACM 1-59593-994-2/05/0007 ...\$5.00.

\section{Categories and Subject Descriptors}

F.2 [Theory of Computation]: Analysis of Algorithms and Problem Complexity; G.2.2 [Mathematics of Computing]: Discrete Mathematics - graph theory, network problems; I.2.11 [Computing Methodologies]: Artificial Intelligence-Distributed artificial intelligence

\section{General Terms}

Performance, Design, Economics

\section{Keywords}

distributed network design, price of anarchy, game-theoretic models, Nash equilibrium, pairwise stability

\section{INTRODUCTION}

Many network settings involve strategic peer selection by independent agents which can be modeled game-theoretically as network formation. These networks are formed endogenously by the actions of agents selfishly maximizing an individual objective function. For this reason, the overall efficiency of such networks, which are the stable outcomes of decentralized strategic interactions, can be worse than the network(s) formed by a central authority maximizing aggregate utility. Understanding this tension between stability and efficiency lies at the heart of our investigation into network formation.

Koutsoupias and Papadimitriou [16, 20] coin the term price of anarchy to refer to the increase in cost caused by independent selfish behavior with respect to the centralized, social welfare-maximizing solution. The price of anarchy has received much attention in games dealing with various networking issues, such as load balancing [7, 21], routing [20], and flow control [9]. This paper is part of a growing body of literature $[10,3,2,6]$ that studies the price of anarchy in network design, where individuals decide whether or not to form links in a shared network.

In many networking applications agents' interactions are regulated by an intermediary. For this reason, we are interested in how the rules governing the network formation process affect the game's equilibrium outcomes. We study how the quality of worst-case and average-case equilibrium networks differ between a network formation game where link creation requires bilateral consent as compared to a setting where links can be established unilaterally. We view the distinction between bilateral and unilateral formation as something that can be controlled through rules enforced by an intermediary. 
The network design game that we study is based on a communication network model by Fabrikant et al. [10] in which agents are nodes and their strategic choices create an undirected graph. We augment the model by having links formed bilaterally (instead of unilaterally) and having the cost of links shared equally between participating nodes. The incorporation of consent represents a natural, realistic extension for a model where traffic routing costs are incurred at both ends of a link.

The bilateral connection game (BCG) is described as follows. Each node simultaneously chooses a (possibly empty) subset of the other nodes in the game, and a link between any two nodes is established if it has the consent of both parties directly involved in the link. The intersection of these sets of edges is the resulting graph. The edges are undirected, meaning that they can be used to route traffic in both directions. Payoffs are construed as costs, meaning that agents are trying to minimize their respective costs of participating in the network, and the cost incurred by an agent is expressed as a trade-off between the cost of establishing links to other agents and its proximity to the rest of the network. We refer to the model by Fabrikant et al. [10] as the unilateral connection game (UCG). It differs from ours in that agents can unilaterally establish links and in the requisite solution concepts for the different models' evaluation. ${ }^{1}$

\subsection{Our Results}

The main contributions of this paper are the following: Adopting the concept of pairwise stability in place of Nash equilibrium, we provide an upper and lower bound on the price of anarchy of the bilateral connection game. We provide a partial characterization of the pairwise stable and efficient networks in the bilateral connection game, and give examples of pairwise stable networks that are not achievable in a Nash equilibrium of the unilateral game. We prove that a subset of equilibrium networks in the unilateral game are pairwise stable in the bilateral game (for the same link cost $\alpha$ ), and conjecture that this holds for all equilibrium networks.

We conduct an empirical analysis that demonstrates that the average price of anarchy is better in the bilateral connection game than in the unilateral game for small link costs but worse as links become more expensive. We explain this by observing that the bilateral setting leads to networks with more links, on average. Along the way, we show a relationship between pairwise stability and a two-player coalitional refinement of Nash called pairwise Nash, as well as a noncooperative solution concept termed a proper equilibrium. Working with pairwise stability is helpful in developing a partial geometric characterization of equilibrium networks in the bilateral connection game. The approach illustrates how the correspondence between different solution concepts can be leveraged to gain structural insight into equilibrium graphs.

The rest of the paper takes the following form: We first discuss related work, before providing a formal introduction to connection games in Section 2. Section 3 deals with the equilibrium and network stability notions that underly much of our analysis. In Section 4, we provide our main results characterizing and comparing the set of stable and efficient network graphs in the unilateral and bilateral connection

\footnotetext{
${ }^{1}$ The incorporation of consent forces us to use an alternative solution concept to analyze stable networks.
}

games. Section 5 presents empirical analysis comparing the two games. Section 6 concludes.

\subsection{Related Work}

Fabrikant et al. [10] study a network creation game in the context of communication networks where links are generated by the unilateral actions of players and link costs are one-sided. Our bilateral network creation model may be better suited for modeling communication network design, given that interconnect costs are typically two-sided.

There is a growing interest in understanding how simple rules of engagement established by a system designer will affect the functioning of a networked system maintained endogenously by the strategic actions of participants [13]. In order to understand the role of bilateral consent in strategic network formation, we compare equilibria and bounds on the price of anarchy in our bilateral network creation game with the unilateral model proposed by Fabrikant et al. [10].

Anshelevich et al. [3, 2] study a different cost-sharing network connection game where, given an undirected graph structure $G$, players have a set of specified terminal nodes that they would like to see connected in the purchased network (which is necessarily a subgraph of $G$ ). In [2], they study how fair cost allocation schemes affect the quality of the best Nash equilibrium network, and so understand the protocol constraining agents' interactions as suggesting an equilibrium outcome. Our model includes only local costsharing and our interaction protocol is restricted to a rule for how individual links are formed and severed, and does not propose a specific equilibrium selection rule. Of mention is that the welfare optimal solution is stable for both connection games we consider.

Lopez-Pintado [17] and Melendex-Jimenez [18] both consider bilateral network formation models with local cost sharing, but their models differ from our model in the payoff structure and the equilibrium concepts applied. Payoffs in both these models are link-separable and derived from underlying (anti-)coordination games, while the benefits of connections in our bilateral game are formulated in terms of a QoS measure based on the entire network topology. Also, both papers use the Nash equilibrium solution concept whereas we rely on the pairwise Nash equilibrium and pairwise stability.

Kannan et al. [15] investigate the effects on network architecture of different distance-based utility functions, but investigate the problem from the standpoint of Nash networks (as developed by Bala and Goyal [4]) and only consider unilateral link costs. Finally Haller and Sarangi [11] provide characterizations of stable networks under one-sided and two-sided link formation costs for a class of link-based payoffs. We are concerned with a different payoff structure, provide a stronger characterization of stable networks for our specific domain, and focus on the price of anarchy.

\section{THE BILATERAL AND UNILATERAL CONNECTION GAMES}

In this section, we present two versions of the Connection Game discussed in this paper: the Unilateral Connection Game (UCG) by Fabrikant et al. [10] and a modified version of the game, including equal-split bilateral link cost shares and mutual consent in link formation, that we call the Bilateral Connection Game (BCG). 
Both games have a finite set of players $N=\{1, \ldots, n\}$. The strategy space of player $i \in N$ is the list of other players, i.e. the set $S_{i}=\left\{\left(s_{i j}\right)_{j \neq i} \mid s_{i j} \in\{0,1\}\right\}$ where $\left|S_{i}\right|=2^{n-1}$. Player $i$ seeks contact with player $j$ if $s_{i j}=1$. Players simultaneously announce the list of other players with whom they wish to be connected. Their decisions generate an undirected graph $G(s)=(N, A(s))$ as per the linking rule of the game. This is a single-stage game with simultaneous announcements.

In the UCG, $A(s)=\left\{(i, j): i \neq j, s_{i j}=1 \vee s_{j i}=1\right\}$. Therefore, a link $(i, j)$ is formed if either participant in the link decides to establish the connection. In the BCG, on the other hand, $A(s)=\left\{(i, j): i \neq j, s_{i j}=1 \wedge s_{j i}=1\right\}$. That is, both players $i$ and $j$ must agree to establish a link in order for it to be created. The only difference between the two games in the creation of links is this issue of consent.

The cost incurred by player $i$ when all players adopt strategy $s$ is additive in the cost of the number of connections $\left|s_{i}\right|$ that player $i$ establishes (or wishes to establish) with other agents, as well as in the sum of the costs of reaching all other agents. Let $\alpha>0$ denote the link cost. The cost to player $i$ is:

$$
c_{i}(s)=\alpha\left|s_{i}\right|+\sum_{j \in N} d_{(i, j)}(G(s))
$$

where $d_{(i, j)}(G(s))$ is the shortest-path distance (in vertex hops) between nodes $i$ and $j$ in the graph $G(s)$. If no path exists between $i$ and $j$ then $d_{(i, j)}(G(s))=\infty$.

The model represents a network setting in which links are costly but good connectivity is desirable. In both the unilateral and bilateral connection games, players seek to minimize their costs (1). While the cost function implies a cost in the BCG for provisioning for links that may not actually form, this cost $\alpha$ without a corresponding edge will not be incurred in equilibrium.

\section{NETWORK EQUILIBRIUM CONCEPTS}

In order to characterize the structure of networks resulting from the network formation games discussed above we need to define what constitutes an equilibrium.

When networks arise from the unilateral action of players, as in the UCG, standard Nash equilibrium analysis can be informative about the structure of networks that emerge. Let $s=s_{N}=\left(s_{i}, s_{N \backslash i}\right)$ and let $\zeta$ designate the set of all undirected networks on players $N$.

Definition 1 (NAsh EQ.). A graph $G(s) \in \zeta$ is a Nash equilibrium network if there exists a strategy $s$ that supports $G(s)$ where $c_{i}(s) \leq c_{i}\left(s_{i}^{\prime}, s_{N \backslash i}\right)$ for all $i \in N$ and $s_{i}^{\prime} \in S_{i}{ }^{2}$

This definition is valid for both the BCG and UCG. However, when network links require the consent of both parties, as in the BCG, a coordination problem arises: the game displays a multiplicity of Nash equilibria stemming from players' capacity for mutual blocking in the formation of links.

For example, the empty network is always a Nash equilibrium when every agent refuses to establish any link. Put another way, an equilibrium concept that only accounts for

\footnotetext{
${ }^{2}$ We restrict our attention to pure-strategy equilibria for two reasons: pure Nash equilibria are guaranteed to exist, and the network stability notion of pairwise stability that we deal with throughout this paper is defined deterministically.
}

single player deviations is insufficient; an appropriate equilibrium concept must consider coalitional moves. A Nash equilibrium outcome that satisfies the additional requirement that it is stable to all bilateral deviations is referred to as a pairwise Nash equilibrium.

In the specific context of our network formation game, a pairwise Nash network is one that contains every mutually beneficial connection given the current graph but no more. In considering only bilateral moves, this is the minimal coalitional refinement of Nash required by games with consent and as such captures the minimal level of coordination amongst players. We further justify this solution concept by providing conditions under which pairwise Nash equilibria in our game correspond to an uncoordinated equilibrium this is robust to small perturbations.

For BCG, it is helpful to define the $n$-square matrix $\Lambda_{(i, j)}$, in which all entries are zero except for $\lambda_{i j}=\lambda_{j i}=1$. This represents a strategy matrix (with row $i$ corresponding to the strategy of player $i$ ) that yields a graph with link $(i, j)$. For the UCG, matrix $\Lambda_{(i, j)}$ is defined as the $n$-square matrix in which all entries are zero except for $\lambda_{i j}=1$. By an abuse of notation, we will write $\Lambda_{B}$ for edge set $B$ to designate the strategy matrix that yields a graph with all links $(i, j) \in B$. Whether this is the strategy matrix for a BCG or UCG game will be clear by context.

Definition 2 (PAirwise NAsh). A graph $G(s) \in \zeta$ is a pairwise Nash equilibrium network if there exists a strategy $s$ that supports $G(s)$ as a Nash equilibrium, and for all $(i, j) \notin A(s)$, if $c_{i}\left(s+\Lambda_{(i, j)}\right)<c_{i}(s)$ then $c_{j}\left(s+\Lambda_{(i, j)}\right)>$ $c_{j}(s)$.

This definition is valid for both the BCG and UCG. The set of Nash equilibria and pairwise Nash equilibria coincide in the UCG, since $c_{i}\left(s+\Lambda_{(i, j)}\right) \geq c_{i}(s)$ is true for any Nash equilibrium.

Following Jackson and Wolinsky [14], we also define the following network stability concept for the BCG:

Definition 3 (PAirwise stable). A graph $G(s) \in \zeta$ is pairwise stable in the $B C G$ if for all $(i, j) \in A(s), c_{i}(s-$ $\left.\Lambda_{(i, j)}\right) \geq c_{i}(s)$, while for all $(i, j) \notin A(s)$, if $c_{i}\left(s+\Lambda_{(i, j)}\right)<$ $c_{i}(s)$ then $c_{j}\left(s+\Lambda_{(i, j)}\right)>c_{j}(s)$.

Let $\Gamma_{\mathrm{PS}} \subset \zeta$ denote the set of pairwise stable networks on $N$ players. The notion of pairwise stability captures a network's stability to the deletion and addition of a single link. To be present, a link must be mutually (weakly) profitable for both players at either end of the link. A link that is missing from the graph, but profitable for one player at the end of the link, must be unprofitable for the other player. In this sense, link severance is unilateral, while link creation is bilateral.

Pairwise stability is a network stability concept that is based on an edge-by-edge analysis of the network rather than an analysis of player strategies (as in a Nash equilibrium). The concept is appealing because it can generate sharp predictions about the tension between stability and efficiency in many contexts [14], and because it makes it easier to characterize the topology of equilibrium networks.

In fact, in the BCG the two solution concepts coincide. That a pairwise Nash equilibrium is pairwise stable is immediate because the two concepts are identical under the addition of links, while pairwise stability only allows an agent to consider the deletion of a single link at a time while pairwise Nash allows for the deletion of any number of links. To 
prove the other direction we leverage a result about convex games due to Calvó-Armengol and Ilkiliç [5]. For this, let $\Gamma \subseteq \zeta$ denote a subset of all undirected networks.

Definition 4 (Convex Costs). A cost function $c_{i}$ is convex on $\Gamma$ if for all s s.t. $G(s)=(N, A(s)) \in \Gamma$, all $i \in N$, and $B \subseteq\{(i, j) \in A(s)\}$ we have:

$$
c_{i}\left(s-\Lambda_{B}\right)-c_{i}(s) \geq \sum_{p \in B} c_{i}\left(s-\Lambda_{p}\right) .
$$

Convexity on set $\Gamma$ implies that the joint marginal value of a group of links in the network is greater than the sum of the marginal values of each link, for all strategy profiles $s$ that correspond to a network in $\Gamma$.

Proposition 1. A graph $G(s) \in \zeta$ for strategy profile $s$ is pairwise stable in the $B C G$ if and only if it is a pairwise Nash equilibrium.

We use the following lemma to prove the result.

LEMMA 1. The cost function $c_{i}$ for the $B C G$ is convex on all graphs $G \in \zeta .^{3}$

Proof. Recall $G(s)=(N, A(s))$. Let $(i, j) \in A(s)$. Note that $d_{(i, k)}(G(s)) \leq d_{(i, k)}\left(G\left(s-\Lambda_{(i, j)}\right)\right), \forall k \in N$. Let

$$
\Delta_{(i, j)}(s)=\left\{k \in N: d_{(i, k)}(G(s))<d_{(i, k)}\left(G\left(s-\Lambda_{(i, j)}\right)\right)\right\} .
$$

In particular, we always have $j \in \Delta_{(i, j)}(s)$. Then $c_{i}\left(s-\Lambda_{(i, j)}(s)\right)=$

$$
\sum_{k \in \Delta_{(i, j)}(s)}\left[d_{(i, k)}\left(G\left(s-\Lambda_{(i, j)}\right)\right)-d_{(i, k)}(G(s))\right]-\alpha .
$$

Let $(i, l) \in A(s)$, where $l \neq j$ and $\Delta_{\{(i, j),(i, l)\}}(s)=$

$$
\left\{k \in N: d_{(i, k)}(G(s))<d_{(i, k)}\left(G\left(s-\Lambda_{\{(i, j),(i, l)\}}\right)\right)\right\} .
$$

Then $c_{i}\left(s-\Lambda_{\{(i, j),(i, l)\}}(s)\right)=$

$\sum_{k \in \Delta_{\{(i, j),(i, l)\}}(s)}\left[d_{(i, k)}\left(G\left(s-\Lambda_{\{(i, j),(i, l)\}}\right)\right)-d_{(i, k)}(G(s))\right]-2 \alpha$.

Consider the following. First,

$$
\left\{\Delta_{(i, j)}(s) \cup \Delta_{(i, l)}(s)\right\} \subset \Delta_{\{(i, j),(i, l)\}}(s) .
$$

Indeed, for all $k \in \Delta_{(i, j)}(s)$, we have

$d_{(i, k)}(G(s))<d_{(i, k)}\left(G\left(s-\Lambda_{(i, j)}\right)\right) \leq d_{(i, k)}\left(G\left(s-\Lambda_{\{(i, j),(i, l)\}}\right)\right)$.

Identically for $\Delta_{(i, l)}(s)$. Second, $\Delta_{(i, j)}(s) \cap \Delta_{(i, l)}(s)=\emptyset$. To see this, let $k \in \Delta_{(i, j)}(s) \cap \Delta_{(i, l)}(s)$. Then both $l$ and $j$ are on two different shortest paths in $G(s)$ between $i$ and $k$. Consider the shortest path in $G(s)$ between $i$ and $k$ that crosses through $l$. This path still exists in $G\left(s-\Lambda_{(i, j)}(s)\right)$, implying that $d_{(i, k)}\left(G\left(s-\Lambda_{(i, j)}\right)\right)=d_{(i, k)}(G(s))$, in contradiction with $k \in \Delta_{(i, j)}(s)$. Third,

$$
\begin{aligned}
& d_{(i, k)}\left(G\left(s-\Lambda_{\{(i, j),(i, l)\}}\right)\right)-d_{(i, k)}(G(s)) \\
\geq & d_{(i, k)}\left(G\left(s-\Lambda_{(i, j)}(s)\right)\right)-d_{(i, k)}(G(s)),
\end{aligned}
$$

\footnotetext{
${ }^{3}$ This proof is based on a result by Calvó-Armengol and Ilkiliç [5], who established convexity for the connections model [14].
}

because

$$
d_{(i, k)}\left(G\left(s-\Lambda_{(i, j)}(s)\right)\right) \leq d_{(i, k)}\left(G\left(s-\Lambda_{\{(i, j),(i, l)\}}\right)\right) .
$$

So too for $d_{(i, k)}\left(G\left(s-\Lambda_{(i, l)}(s)\right)\right)-d_{(i, k)}(G(s))$. Altogether, this implies that:

$c_{i}\left(s-\Lambda_{\{(i, j),(i, l)\}}(s)\right) \geq c_{i}\left(s-\Lambda_{(i, j)}(s)\right)+c_{i}\left(s-\Lambda_{(i, l)}(s)\right)$.

This proves that cost function $c_{i}$ is convex.

We now show that convexity means that pairwise stability implies a network is a pairwise Nash equilibrium. By pairwise stability, no player has an incentive to sever any single link in a unilateral deviation, and thus no player has an incentive to sever multiple links by convexity. This satisfies the pairwise Nash requirement in regard to the effect of a player's strategy on existing edges. In considering the effect of some strategy $s_{i}^{\prime} \neq s_{i}$ on adding links, the requirement that strategy $s$ be a Nash equilibrium is vacuous because $s_{j i}=0$ for any $s_{i j}=0$, and so a unilateral deviation can only incur cost without creating an edge. The remaining requirement in regard to cooperatively adding a single, missing link is the same for pairwise stable and pairwise Nash.

Still, the concept of a pairwise Nash equilibrium allows explicit player coordination in regard to adding a link. We can gain further justification for this equilibrium concept by establishing a relationship between pairwise Nash networks and networks formed in a proper equilibrium [19].

Definition 5 (Proper EQUilibrium). A pure strategy profile $\sigma \in \Sigma$ is proper if there exists a sequence $\left\{\epsilon_{t}\right\}_{t \in \mathbb{N}}$ of positive real numbers converging to zero and a sequence of completely mixed equilibrium strategy profiles $\left\{\sigma^{\epsilon_{t}}\right\}_{t \in \mathbb{N}}$ with limit $\sigma$ such that, for all $i \in N$, all $s_{i}^{\prime}, s_{i}^{\prime \prime} \in S_{i}$, and all $t \in \mathbb{N}$, we have:

$E\left[c_{i}\left(s_{i}^{\prime}, \sigma_{N \backslash i}^{\epsilon_{t}}\right)\right]<E\left[c_{i}\left(s_{i}^{\prime \prime}, \sigma_{N \backslash i}^{\epsilon_{t}}\right)\right]$ implies $\sigma_{i}^{\epsilon_{t}}\left(s_{i}^{\prime \prime}\right) \leq \epsilon_{t} \cdot \sigma_{i}^{\epsilon_{t}}\left(s_{i}^{\prime}\right)$.

In a proper equilibrium, players play a best response to perturbations of their opponents' strategies, where costly mistakes are made with less probability. This is a noncooperative refinement of Nash equilibrium and does not require any coordination on the part of players. Every game has a proper equilibrium in mixed strategies [19].

Using a result by Calvó-Armengol and Ilkiliç [5], we establish that all pairwise Nash equilibrium networks in the BCG that satisfy a convexity property are proper equilibria. For this, we introduce an additional notion of convexity, which is stated for link addition and deletion.

Definition 6 (Link CONVEXity). A graph $G(s)=$ $(N, A(s)) \in \zeta$ is link convex if for all $(i, k) \notin A(s)$, and all $(l, m) \in A(s)$,

$$
\begin{aligned}
& \sum_{j} d_{(i, j)}(G(s))-\sum_{j} d_{(i, j)}\left(G\left(s+\Lambda_{(i, k)}\right)\right) \\
< & \sum_{j} d_{(l, j)}\left(G\left(s-\Lambda_{(l, m)}\right)\right)-\sum_{j} d_{(l, j)}(G(s)) .
\end{aligned}
$$

Considering the effect on distances, this says that the decrease in cost to agent $i$ from adding a missing link $(i, k)$ is less than the increase in cost to agent $l$ from removing an existing link $(l, m)$. Link convexity is a sufficient condition for a graph to be pairwise stable for some link cost $\alpha$.

Lemma 2. A graph $G(s)=(N, A(s)) \in \zeta$ is pairwise stable for some link cost $\alpha$ if the graph is link convex. 
Proof. Graph $G(s)$ is pairwise stable for link cost $\alpha$ if and only if $\alpha \in\left(\alpha_{\min }, \alpha_{\max }\right]$, where $\alpha_{\min }=$

$$
\begin{aligned}
& \max _{(i, k) \notin A(s)} \min \left\{\sum_{j} d_{(i, j)}(G(s))-\sum_{j} d_{(i, j)}\left(G\left(s+\Lambda_{(i, k)}\right)\right),\right. \\
&\left.\sum_{j} d_{(k, j)}(G(s))-\sum_{j} d_{(k, j)}\left(G\left(s+\Lambda_{(i, k)}\right)\right)\right\},
\end{aligned}
$$

and $\alpha_{\max }=$

$$
\min _{(l, m) \in A(s)}\left\{\sum_{j} d_{(l, j)}\left(G\left(s-\Lambda_{(l, m)}\right)\right)-\sum_{j} d_{(l, j)}(G(s))\right\} .
$$

To understand this characterization of pairwise stability, note that for link addition, we only require that the link cost $\alpha$ is too high for the least-interested agent involved in the link, i.e. the agent who incurs the least benefit from the new link $(i, k)$. Then $\alpha$ must be more expensive than the largest cost savings to any of these least-interested agents (this is $\left.\alpha_{\min }\right)$, since otherwise $i$ and $k$ would bilaterally establish that link.

On the other hand, $\alpha$ can be no bigger than the minimum reduction in distance costs that any player, say $l$, would achieve by severing some link to $m$ (this is $\alpha_{\max }$ ), otherwise $l$ will unilaterally opt to sever that link.

Link convexity is a sufficient property for the existence of a link cost $\alpha$ for which $G(s)$ is pairwise stable. This is because $\alpha_{\min } \leq$

$$
\begin{gathered}
\max _{(i, k) \notin A(s)}\left\{\sum_{j} d_{(i, j)}(G(s))-\sum_{j} d_{(i, j)}\left(G\left(s+\Lambda_{(i, k)}\right)\right)\right\}< \\
\min _{(l, m) \in A(s)}\left\{\sum_{j} d_{(l, j)}\left(G\left(s-\Lambda_{(l, m)}\right)\right)-\sum_{j} d_{(l, j)}(G(s))\right\},
\end{gathered}
$$

where the weak inequality is by the definition of $\alpha_{\min }$, and the strict inequality follows by the definition of link convexity.

We now appeal to the following result, which holds when neither agent on either end of a link $(i, j)$ absent from $G(s)$ would consent to the addition of the link:

Lemma 3 (CAlvó-Armengol And Ilkiliç [5]). A pairwise Nash network $G(s)=(N, A(s)) \in \zeta$ for link cost $\alpha$ where, for any edge $(i, j) \notin A(s)$ then $c_{i}\left(s+\Lambda_{(i, j)}\right)>c_{i}(s)$, is a proper equilibrium for the same link cost.

Proposition 2. A graph $G(s)=(N, A(s)) \in \zeta$ is achievable as a proper equilibrium of the $B C G$ for some link cost $\alpha$ if the graph is link convex.

Proof. By link convexity, there exists some link cost $\alpha$ such that no agent on either end of a missing link can benefit from adding the link (3). The result follows by Lemma 3, noting that this graph is also a pairwise stable network by Lemma 2, and a pairwise Nash network by Proposition 1.

Going forward, we adopt the simple notion of pairwise stability to characterize the set of equilibrium topologies in the BCG and study the price of anarchy of the BCG.

\section{STABILITY AND EFFICIENCY OF NETWORKS IN THE BCG}

In this section we address the question of what pairwise stability predicts concerning the graphs that might form in the BCG. We proceed as follows. We first characterize the set of efficient graphs and provide a partial characterization of topologies that are stable in the bilateral game, with a particular focus on graphs which are not achievable in a Nash equilibrium of the UCG in order to show the difference between the outcomes of the two games. We then prove an upper and lower bound on the price of anarchy in the BCG. The theoretical analysis in this section is complemented with an empirical analysis in Section 5, where we study the average price of anarchy of stable networks in both the BCG and UCG.

In discussing the efficiency of a graph we appeal to the notion of the social cost of a graph. By an abuse of notation we let $C(G)$ denote the social cost for the graph $G(s)=$ $(N, A(s))$. The social cost of a network $G(s)$ in the BCG is the sum of all players' costs,

$$
C(G)=\sum_{i \in N} c_{i}(s)=2 \alpha|A(s)|+\sum_{i, j \in N} d_{(i, j)}(G(s)) .
$$

The efficient graph is the graph that minimizes the social cost. Noting that every pair of non-neighboring vertices is at least distance two apart, the following is a lower bound for the social cost:

$$
\begin{aligned}
C(G) & \geq 2 \alpha|A(s)|+2|A(s)|+2(n(n-1)-2|A(s)|) \\
& =2 n(n-1)+2(\alpha-1)|A| .
\end{aligned}
$$

The bound is met by any graph $G(s)$ of diameter two.

Lemma 4. For the BCG and link cost $\alpha<1$, the complete graph is the only efficient graph and the only pairwise stable graph.

Proof. To prove the first statement, by (5), the social optimum is achieved when $|A|$ is maximum. Moreover, consider $s^{\prime}=s+\Lambda_{(i, j)},(i, j) \notin A(s)$. Then $c_{k}\left(s^{\prime}\right)=c_{k}(s)+\alpha-$ $1<c_{k}(s), k=\{i, j\}$ and $c_{k}\left(s^{\prime}\right) \leq c_{k}(s), k \in N \backslash\{i, j\}$. For the second statement, note that any stable graph must be of diameter 1 , since the addition of any new link will reduce inter-node distances by at least $1>\alpha$.

For link cost $\alpha<1$, there is no conflict between stability and efficiency. The situation changes for $\alpha>1$. This is true in both the BCG and UCG. Indeed, many network formation models $[10,13]$ exhibit such a transition.

Lemma 5. For link cost $\alpha>1$, the star network is the only efficient graph in the BCG. The star network is also pairwise stable, but one of many stable graphs.

Proof. For the first statement, the total cost of $k$ indirect links in a graph $G$ is $2 k$. For $\alpha>1$, the cost from a direct link exceeds the cost from an indirect link of length two. So direct links must be minimized, as (5) suggests, and the length of all indirect connections must be two. On the other hand, the connectedness of $G$ requires at least $n-1$ direct connections. Given these conditions, the star is the only efficient network. To prove that the star is pairwise stable, note that no two non-adjacent agents have any incentive to link directly in a star while no agent wants to disconnect themselves from the graph. That the star is not 
unique is proven by the different stable topologies that are mentioned in the discussion that follows (Lemma 6).

The non-uniqueness of the socially optimal graph amongst pairwise stable graphs for link cost $\alpha>1$ is what motivates our analysis of the cost of the worst-case equilibrium (otherwise called the price of anarchy) of the BCG. The network is formed endogenously solely by the actions of players, and so this worst case scenario cannot be guarded against.

The price of anarchy $\rho(\geq 1)$ of a network $G$ is defined as follows: $\rho(G)=C(G) / \min _{G^{\prime} \in \zeta} C\left(G^{\prime}\right)$. In words, this is the ratio of social cost of the graph $G$ to the social cost of the efficient graph.

The price of anarchy of the BCG is defined as the ratio of social costs of the highest cost pairwise stable graph to the efficient graph:

$$
\rho_{\mathrm{BCG}}=\max _{G \in \Gamma_{\mathrm{PS}}} \rho(G),
$$

where $\Gamma_{\mathrm{PS}} \subset \zeta$ is the set of pairwise stable networks.

For $\alpha>1$, the efficient graph is the star graph, and the price of anarchy of a network $G$ with edges $A$ is:

$$
\rho(G)=\frac{2 \alpha|A|+\sum_{i, j} d_{(i, j)}(G)}{2 \alpha n+2 n(n-1)}
$$

We now identify some graphs that are pairwise stable for this range of link costs:

Lemma 6. The cycle $C_{n}$ is pairwise stable in the $B C G$ for some link cost $\alpha>1$, and has price of anarchy $\rho\left(C_{n}\right)=$ $O(1)$.

Proof. (Sketch) By link convexity, $C_{n}$ is pairwise stable if $\frac{n^{2}-4 n+4}{8}<\alpha<\frac{n(n-2)}{4}$ for $n=4 k-2, \frac{n^{2}-4 n+8}{8}<\alpha<$ $\frac{n(n-2)}{4}$ for $n=4 k, \frac{(n-3)(n+1)}{8}<\alpha<\frac{(n+1)(n-1)}{4}$ for $n=$ $2 k-1$, all for $k \in \mathbb{N}$. Then $\alpha=O\left(n^{2}\right)$, and $\rho\left(C_{n}\right)=$ $O\left(2 \alpha n+n^{3}\right) /\left(2 \alpha n+2 n^{2}\right)=O\left(n^{3} / n^{3}\right)=O(1)$.

We can also show that all strongly regular graphs (see Figure 1), where all adjacent vertices have $\lambda>0$ common neighbors, and all non-adjacent vertices have $\mu>1$ common neighbors are pairwise stable. These graphs also have price of anarchy $O(1)$. The proof is omitted for lack of space. There are, however, more costly, pairwise stable networks in the $\mathrm{BCG}$.

\subsection{A Lower Bound on the Price of Anarchy}

We establish a lower bound on the price of anarchy of the BCG by considering the pairwise stability of a class of regular graphs whose order (the size of the graph) is a constant factor of the Moore bound [12]. The Moore bound establishes an upper limit $n=\left(k(k-1)^{D}-2\right) / 2$ on the number of nodes $n$ of a regular graph $G_{k, D}$ of degree $k$ and diameter $D$.

Proposition 3. The worst-case price of anarchy in the $B C G$ is $\Omega\left(\log _{2} \alpha\right)$ for link cost $\alpha$.

To prove this we make use of the following lemma.

Lemma 7. All regular graphs $G_{k, D}$ (for degree $k$ and $d i$ ameter D) whose order is a constant factor of the Moore bound are pairwise stable for some link cost $\alpha$, and have price of anarchy $\Omega\left(\log _{2} \alpha\right)$.
Proof. (Sketch) We first establish that such a graph is link convex. Consider a $k$-regular graph with shortest cycle (girth) $g$. The removal of any link will increase the distance of one vertex to $g-1$, of $k-1$ vertices to $g-2$, and so on for all vertices along a path up to $g / 2$ away, leading to total increase in distance cost of: $S_{r}=\sum_{i=1}^{g / 2}(k-1)^{i+1}(g-i)$. On the other hand, the best-case addition for any link is on the opposite side of a shortest cycle. The addition of such a link will reduce the distance to nodes along two tree paths of depth $g / 4$. That is, the distance cost decrease from adding any link is upper bounded by $S_{a}=\sum_{i=1}^{g / 4}(k-1)^{i+1}(g-$ $i)$. Clearly $S_{r}>S_{a}$, which establishes link convexity, and ensures pairwise stability for some $S_{a}<\alpha<S_{r}$.

We now establish the price of anarchy of such a graph. Consider a generalized Moore polygon, such that from any vertex the number of nodes at distance $0,1,2, \ldots, D$ is $1, r, r(r-$ $1), r(r-1)^{2}, \ldots$ with the remaining vertices necessarily in the furthest position, and where $D$ is the diameter of the graph. This assumption is not restrictive since only the highest power matters to establish order, which does not change with this particular construction. Then we have:

$$
\begin{gathered}
C\left(G_{k, D}\right)=n\left(\alpha k+k \sum_{i}^{D-1} i(k-1)^{i-1}+\right. \\
\left.\left(n-1-k \sum_{i}^{D-1} i(k-1)^{D-1}\right) D\right) \\
\geq n \alpha k+n k \sum_{i}^{D-1} i(k-1)^{i-1},
\end{gathered}
$$

where the order of the graph is a fixed constant times the Moore bound of $\frac{k(k-1)^{D}-2}{2}$. Moreover, the increase in distance cost from the removal of any link can be shown to be lower bounded by $\sum_{i=1}^{D} i\left(2^{D-i}\right)$, expressed now in terms of $D$. Together, we have that $n=\Theta\left(k^{D}\right)$ and $\alpha=\Theta\left(2^{D}\right)$. For sufficiently large $n$, the price of anarchy becomes $\rho\left(G_{k, D}\right)=$ $\Omega(D)=\Omega\left(\log _{2} \alpha\right)$.

This establishes a lower bound on the price of anarchy in the BCG of $\rho_{\mathrm{BCG}}=\Omega\left(\log _{2} \alpha\right)$.

The result also establishes that extremal graphs, which are the largest $k$-regular graphs of diameter $D$, and cage graphs, which are the smallest $k$-regular graphs of girth $g$, are pairwise stable for some $\alpha{ }^{4}$ Extremal graphs and cage graphs feature prominently in network design since they have small diameter and exhibit high fault tolerance for nodes with specified degree [5] (see Figure 1). As such, it is a good feature that they appear as stable networks in the BCG. It is also worth mentioning that these graphs are generally not achievable in a Nash equilibrium of the UCG.

The condition of link convexity, although only sufficient and not necessary in general, tends to be effective in delineating which graphs are pairwise stable. In particular, many

\footnotetext{
${ }^{4} \mathrm{By}$ the same argument, we believe that these graphs have price of anarchy $\Omega\left(\log _{2} \alpha\right)$. We can prove by the same construction that extremal and cage graphs are link convex. However, the $\Omega\left(\log _{2} \alpha\right)$ bound holds contingent on these graphs being a constant factor from the Moore bound. Proving this for general $k$-regular graphs is an open problem in graph theory, but is widely conjectured to be true.
} 
asymmetric and non-regular graphs that contain cycles are not link convex, and are not pairwise stable. Many symmetric graphs are also inadmissible. For example, while the Desargues graph, a symmetric cubic distance-regular graph on 20 vertices and 30 edges with diameter-to-girth ratio of $2 / 3$ is link convex, the dodecahedral graph, a symmetric cubic planar graph on 20 vertices and 30 edges with a diameterto-girth ratio of 1 is not.
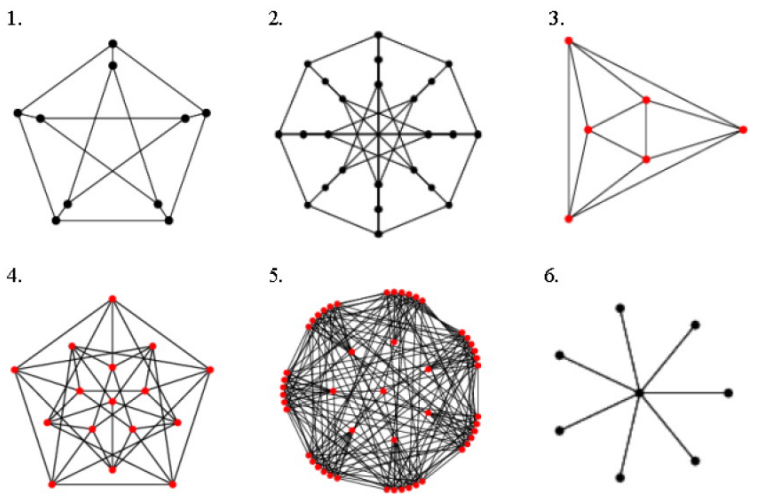

6.



Figure 1: Pairwise stable graphs. 1. The Petersen graph is the unique $(3,5)$ cage and Moore graph, and is strongly regular with parameters $(10,3,0,1) .2$. The McGee graph is a $(3,7)$ cage. 3 . The octahedral graph is strongly regular with parameters $(6,4,2,4)$. 4 . The Clebsch graph is strongly regular with parameters $(16,5,0,2)$. 5. The Hoffman-Singleton graph is the unique $(7,5)$ cage and Moore graph, and is strongly regular with parameters $(50,7,0,1)$. 6 . The star graph on 8 vertices.

\subsection{An Upper Bound on the Price of Anarchy}

We now provide an upper bound on the price of anarchy in the BCG.

Proposition 4. For link cost $\alpha<n^{2}$, the worst-case price of anarchy in the $B C G$ is $O(\sqrt{\alpha})$.

Proof. Note that $d_{(i, j)}(G)<2 \sqrt{\alpha}$ for every $i, j \in N$ since otherwise $i$ and $j$ would bilaterally establish a direct link to bring themselves closer to all nodes more than half way to each other along the shortest path from $i$ to $j$. So we want to show that $|A|=O\left(n^{2} / \sqrt{\alpha}\right)$. For any edge $a_{i}$ out of vertex $v$ we will count all vertices $u$ for which $(v, u) \notin G$. Let $T_{i}=\{u \in N$ : the shortest path from $v$ to $u$ goes through $\left.a_{i}\right\}$. We ensure that $T_{i}$ are disjoint by assuming a canonical shortest path for every vertex. Note that before $a_{i}$ was built, in graph $G^{\prime}$, the alternative shortest path from $v$ to $u \in T_{i}$ either didn't exist or had length at most $2 D(G)<4 \sqrt{\alpha}$, where $D$ is the diameter of $G$. In the first case, the graph has two components and we count $\left|T_{i}\right|-1+$ $\left|V-T_{i}\right|-1=n-2=\Omega(\sqrt{\alpha})$ non-edges (those incident on $v$ or $w$ and the other component, where $\left.a_{i}=(v, w)\right)$. In the second case, $d_{(v, u)}\left(G^{\prime}\right)-d_{(v, u)}(G)<4 \sqrt{\alpha}$. The total improvement is necessarily $\sum_{u}\left(d_{(v, u)}\left(G^{\prime}\right)-d_{(v, u)}(G)\right) \geq \alpha$, i.e. $\left|T_{i}\right|=\Omega(\sqrt{\alpha})$. In either case, non-edges are counted at most twice. Therefore $|A|=n^{2} / \sqrt{\alpha}$ and the price of anarchy is $O((n \sqrt{\alpha}) /(\sqrt{\alpha}+n))$. For $\alpha<n$, this upper bound is $O(\sqrt{\alpha})$; for $\alpha>n$, this upper bound is $O(n / \sqrt{\alpha})$. Combined, the upper bound is $O(\min (\sqrt{\alpha}, n / \sqrt{\alpha}))$, and $O(\sqrt{\alpha})$.
In addition, as pointed out by Demaine et al. [8] after the first version of this paper, the proof shows that the price of anarchy is $O(\min (\sqrt{\alpha}, n / \sqrt{\alpha}))$, which gives the improved bound of $O(n / \sqrt{\alpha})$ for $\alpha>n$.

\subsection{The Price of Anarchy in the BCG vs. the UCG}

In this section, we quantify and compare the price of anarchy in the BCG and UCG. Fabrikant et al. [10] conjectured that all (non-transient) Nash equilibria in the UCG are trees, for some link cost $\alpha>\underline{\alpha}$ for some constant $\underline{\alpha}$. They showed that if this tree conjecture holds, the price of anarchy of the $\mathrm{UCG}$ is constant.

Proposition 5. Let $G(s)$ be a tree and a Nash equilibrium graph of the $U C G$ for some link cost $\alpha$. Then $G(s)$ is pairwise stable in the BCG for the same link cost.

Proof. If $G(s)$ is a Nash equilibrium of the UCG then link cost $\alpha \in C_{\alpha}$, where

$$
\begin{gathered}
C_{\alpha}=\left\{\alpha^{\prime}: \alpha^{\prime}\left(\left|s_{i}^{\prime}\right|-\left|s_{i}\right|\right) \geq\left|T_{i}(s)\right|-\left|T_{i}\left(s-\Lambda_{s_{i}}+\Lambda_{s_{i}^{\prime}}\right)\right|\right. \\
\left.\forall i, \forall s_{i}^{\prime} \in S_{i}\right\} \neq \emptyset
\end{gathered}
$$

We now restrict our attention to the set of deviations by $i$ such that $\left|s_{i}^{\prime}\right|=\left|s_{i}\right|+1$. Let $\Delta_{\left|s_{i}\right|+1}=\left\{r \in S_{i}:|r|=\right.$ $\left.\left|s_{i}\right|+1\right\}$. By (8), for a Nash equilibrium in UCG we need,

$$
\alpha \geq\left|T_{i}(s)\right|-\left|T_{i}\left(s-\Lambda_{s_{i}}+\Lambda_{s_{i}^{\prime}}\right)\right|, \forall i, \forall s_{i}^{\prime} \in \Delta_{\left|s_{i}\right|+1} .
$$$$
\text { Clearly, }\left\{r \in S_{i}: r=s_{i}+\Lambda_{(i, j)}, j \notin A(s)\right\} \subset \Delta_{\left|s_{i}\right|+1} \text {. }
$$

Therefore

$$
\alpha \geq\left|T_{i}(s)\right|-\left|T_{i}\left(s+\Lambda_{(i, j)}\right)\right|, \forall i, \forall(i, j) \notin A(s)
$$

This is also satisfies the lower bound expression $\alpha_{\text {min }}$ for pairwise stability in the BCG (and in particular (3)). Furthermore, if a graph is also stable to link severance because without any edge the graph would be separated into two components. The result follows.

Albers et al. [1] disproved the tree conjecture for the UCG, but were able to give a constant upper-bound on the price of anarchy in the UCG for $\alpha \in O(\sqrt{n})$ and $\alpha \geq 12 n\lceil\log n\rceil$ (for which every Nash equilibrium in the UCG is indeed a tree).

We conjecture that all Nash graphs of the UCG are pairwise stable in the BCG for the same link cost. This conjecture is made on the basis of simulation results and analysis of Nash graphs of the UCG. ${ }^{5}$ If true, then this would establish that the price of anarchy is no better in the BCG than the UCG, whatever the link cost. ${ }^{6}$ Without proving or

\footnotetext{
${ }^{5}$ In addition, not all pairwise stable graphs are Nash. For example, cycle $C_{n}$ for $n>5$ (with each edge incurring the cost of the edge in the clockwise direction), is not Nash supportable in the UCG, since node 0 would lower its cost if it linked to node 2 instead. Yet the cycle is a stable configuration of the BCG (Lemma 6).

${ }^{6} \mathrm{In}$ particular, for $\alpha=f(n), \rho_{\mathrm{UCG}}(n) \in O\left(\rho_{\mathrm{BCG}}(n)\right)$. For $\alpha>2$, the optimum graph in both the UCG and the BCG is a star. Consider any graph $G$ (of size $n$ ) with cost $C$ in
} 


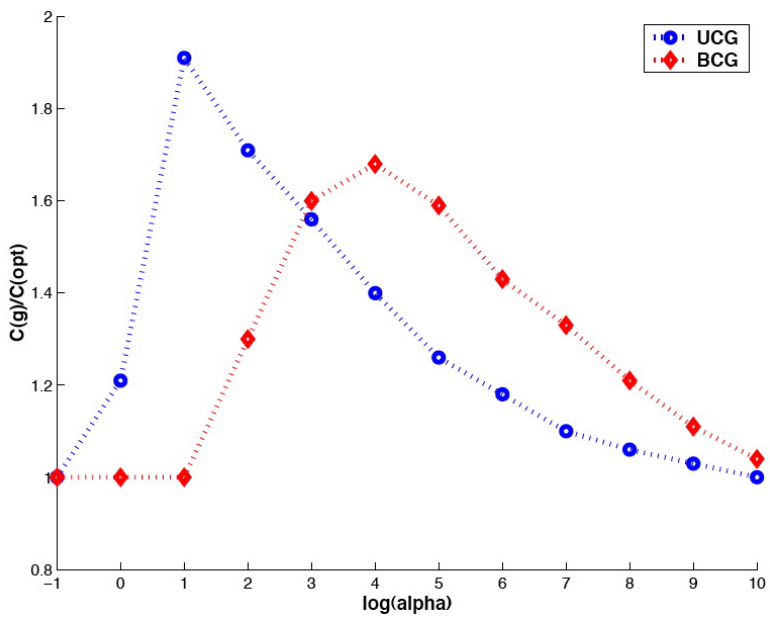

Figure 2: The average PoA in equilibrium networks with ten agents in the UCG and BCG, as a function of link cost. (For link cost $\alpha$, the x-axis plots $\log (\alpha)$ and $\log (2 \alpha)$ in the UCG and BCG, respectively.)

disproving this conjecture, Demaine et al. [8] prove that the price of anarchy is higher in the BCG than the UCG. First, they give improved upper-bounds in the UCG to prove a constant upper bound for $\alpha=O\left(n^{1-\epsilon}\right)$ for any fixed $\epsilon>0$, and obtain an $o\left(n^{\epsilon}\right)$ bound for general $\alpha$; see also Albers et al. [1]. Second, they prove that our upper bound in the BCG is tight, establishing the price of anarchy of the BCG to be $\Omega(\min (\sqrt{\alpha}, m / \sqrt{\alpha}))$. This gives a strict separation between the price of anarchy in the UCG and the BCG.

\subsection{Discussion}

The best-response dynamic in the UCG is more powerful than in the BCG. The problem of mutual blocking in the formation of new links in the BCG and the limited capacity to coordinate afforded to players under pairwise stability means that players are less able to react to the positive externalities of other players' links, which is the root cause of the tension between stability and efficiency in the connection game for $\alpha>1$. This has two major consequences.

On the one hand, the set of pairwise stable network geometries in the BCG is richer than the set of Nash equilibrium network shapes admitted in the UCG. For example, extremal and cage graphs, which feature prominently in many communication network designs, are pairwise stable in the BCG but not generally Nash supportable in the UCG. ${ }^{7}$

the UCG. The cost in the BCG is at least $C+\alpha(n-1)$. Adopting an expression for the social cost of the star in the denominator, we have $\rho_{\mathrm{UCG}}(G)=C /((n-1)(2 n+\alpha-2))$ and $\rho_{\mathrm{BCG}}(G) \geq(C+\alpha(n-1)) /((n-1)(2 n+2 \alpha-2))$. For any $\alpha=$ $f(n), \rho_{\mathrm{UCG}}(G) \leq 2 \rho_{\mathrm{BCG}}(G)$. For $1<\alpha \leq 2$ the complete graph is the optimum in the UCG and the star remains the optimum in the BCG. By a similar analysis, for any graph $G$ with cost $C$ in the UCG we have $\rho_{\mathrm{UCG}}(G)=C /(n(n-$ 1) $((\alpha-2) / 2+2)$ and $\rho_{\mathrm{BCG}}(G) \geq(C+\alpha(n-1)) /((n-$ 1) $(2 n+2 \alpha-2))$. For any $1<\alpha \leq 2, \rho_{\mathrm{UCG}}(G) \leq C /((n-$ 1) $(3 n / 2)) \leq 2 C /((n-1)(2 n+2 \alpha-2)) \leq 2 \rho_{\mathrm{BCG}}(\bar{G})$ for large enough $n$, where the first inequality follows by substituting $\alpha=1$.

${ }^{7}$ With the notable exception of the Petersen graph $((3,5)$ cage), which constitutes a Nash equilibrium of the UCG for

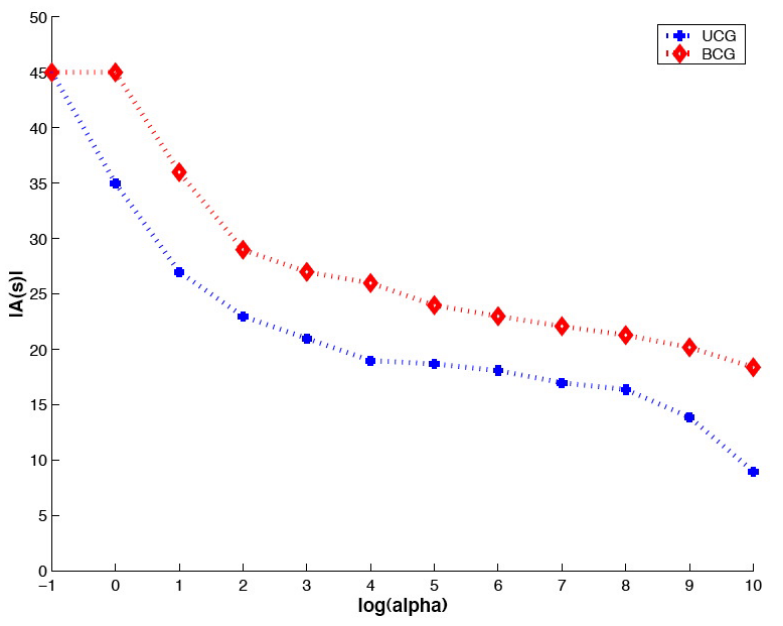

Figure 3: The average number of links in equilibrium networks with ten agents in the UCG and BCG as a function of link cost. (For link cost $\alpha$, the x-axis plots $\log (\alpha)$ and $\log (2 \alpha)$ in the UCG and BCG, respectively.)

On the other hand, the limited capacity of players in the BCG to react to externalities in the game is what drives the social cost of an equilibrium graph in the BCG to be worse than in the UCG.

\section{AN EMPIRICAL STUDY OF THE AVERAGE PRICE OF ANARCHY}

In this section we quantify the difference between the price of anarchy of equilibrium networks in the BCG and the UCG over the set of equilibrium networks, as opposed to only the worst case network. In particular, we want to capture the difference between the average price of anarchy of equilibrium networks in both games for a given link cost $\alpha$. Solving for a Nash network in the UCG and a pairwise stable network in the BCG are both $N P$-complete problems [10, 14, 22]. Given the intractability of providing a full characterization of all equilibrium networks for a large number of players, we restrict our attention to small networks. On the other hand, we want to consider a large enough setting so that the multiplicity of equilibria will bring out the difference between the BCG and UCG. In what follows we consider a setting with ten agents. We compute all pairwise stable graphs in the BCG and all Nash graphs in the UCG by enumeration of all connected topologies on ten vertices. ${ }^{8}$

Figure 2 shows the average price of anarchy of equilibrium networks in the BCG and UCG for different link cost values. The general increase in the average price of anarchy for intermediate link costs is explained by the multiplicity of suboptimal equilibria (all equilibrium networks are trees for $\alpha>n^{2}$ ). In contrast, for intermediate link costs many inefficient topologies are admitted to the stable set (in addition to the efficient graph).

The results corroborate the theoretical analysis, reflecting

$1 \leq \alpha \leq 4$.

${ }^{8}$ The precise algorithm is omitted for lack of space. Our graph enumeration technique hinges on many fast checks to rule out inadmissible topologies before exhaustively checking if they constitute equilibria. 
a stark difference between the topologies of graphs admitted by the BCG and UCG. In addition, we see that when links are inexpensive, the average price of anarchy in the BCG is lower than for the UCG. However, as links become more expensive the situation changes: pairwise stable networks in the BCG are then generally less efficient than Nash networks in the UCG.

Whereas agents in the BCG can get stuck in a badly suboptimal configuration, the ability of agents in the UCG to arbitrarily change their outgoing links means players can construct a smaller number of well-placed links. In contrast, as links become more expensive, stable networks in the BCG tend to become overconnected and increasingly inefficient. Indeed, the plot in Figure 3 shows that stable graphs in the BCG contain more edges than equilibrium graphs in the UCG on average, for a range of link costs. As links become more expensive, graphs with more edges are increasingly over-connected and inefficient.

\section{CONCLUSION}

We presented a bilateral, consent-driven model of network formation. We prove a a lower bound of $\Omega\left(\log _{2} \alpha\right)$ and an upper bound of $O(\sqrt{\alpha})$ on the price of anarchy in the game for pairwise stable networks (equivalently, networks achievable in a pairwise Nash equilibrium.) We conjecture that all Nash graphs of the UCG are pairwise stable in the BCG for the same link cost. This conjecture would establish that the price of anarchy is no better in the BCG than the UCG, whatever the relationship between link cost $\alpha$ and size of graph, $n .^{9}$ Empirical analysis of equilibrium networks in the BCG and the UCG also suggests that the worst-case is strictly worse in the bilateral game. In addition, while the average price of anarchy of equilibrium networks in the bilateral setting is better than in the unilateral setting when links are inexpensive, the situation is reversed when links become more expensive. Altogether, the comparative results suggest that worst-case equilibrium networks have a higher price of anarchy in a consent-driven network formation setting where players are limited in their ability to coordinate simultaneously than when consent is not required and players are unconstrained in their ability to act selfishly.

Our investigations into the role of consent in network formation suggest that the rules of engagement strongly affect the set of possible equilibria. We are currently investigating how bilateral and multilateral transfers between players may help mediate the price of anarchy in the connection game. We are also interested in extending the game to a setting where the network formation is dynamic and on-going. The dynamics of network formation can be controlled by an intermediary, subject to equilibrium constraints suggested by the dynamic network formation process.

\section{Acknowledgments}

We thank Antoni Calvó-Armengol and Rahmi Ilkiliç for helpful discussions, particularly in regard to the convexity of the cost function in the connections game. We also thank Geoff Exoo for helping to generalize the lower bound on the price of anarchy result.

\footnotetext{
${ }^{9}$ Since the paper was published, and without proving or disproving this conjecture, Demaine et al [8] showed that our upper-bound is tight, and established a separation between the price of anarchy in the BCG and the UCG.
}

\section{REFERENCES}

[1] S. Albers, S. Eilts, E. Even-Dar, Y. Mansour, and L. Roditty. On Nash equilibria for a network creation game. In Proc. 17th Ann. ACM-SIAM Symp. on Discrete algorithms (SODA'06), pages 89-98, 2006.

[2] E. Anshelevich, A. Dasgupta, J. Kleinberg, E. Tardos, T. Wexler, and T. Roughgarden. The price of stability for network design with fair cost allocation. In Proc. 45th Ann. IEEE Symp. on Found. of Computer Sci. (FOCS'04), pages 295-304, 2004.

[3] E. Anshelevich, A. Dasgupta, E. Tardos, and T. Wexler. Near-optimal network design with selfish agents. In Proc. 35th Ann. ACM Symp. on Theory of Computing (STOC'03), pages 511-520, 2003.

[4] V. Bala and S. Goyal. A non-cooperative model of network formation. Econometrica, 68(5):1181-1229, 2000.

[5] A. Calvó-Armengol and R. Ilkiliç. Pairwise-stability and Nash equilibria in network formation. Working Papers 2005.34, Fondazione Eni Enrico Mattei, March 2005.

[6] B. Chun, R. Fonseca, I. Stoica, and J. Kubiatowicz. Characterizing selfishly constructed overlay networks. In Proc. 23rd Ann. Joint Conf. of the IEEE Computer and Comm. Societies (INFOCOM'04), pages 1329-1339, 2004.

[7] A. Czumaj and B. Vöcking. Tight bounds for worst-case equilibria. In Proc. 13th Ann. Symp. on Discrete Algorithms (SODA'02), pages 413-420, 2002.

[8] E. D. Demaine, M. Hajiaghayi, H. Mahini, and M. Zadimoghaddam. The price of anarchy in network creation games. In Proc. 26th Ann. ACM Symp. on Princ. of Distr. Comput. (PODC'07), pages 292-298, 2007.

[9] D. Dutta, A. Goel, and J. Heidemann. Oblivious AQM and Nash equilibria. In Proc. 22nd Ann. Joint Conf. of IEEE Comp. and Comm. Societies (INFOCOM'03), pages 106-113, 2003.

[10] A. Fabrikant, A. Luthra, E. Maneva, C. H. Papadimitriou, and S. Shenker. On a network creation game. In Proc. 22nd Ann. Symp. on Princ. of Distributed Computing (PODC'03), pages 347-351, 2003.

[11] H. Haller and S. Sarangi. Nash networks with heterogeneous agents. Mathematical Social Sciences, 50:181-201, 2005.

[12] D. Holton. The Petersen Graph. CUP, 1993.

[13] M. O. Jackson. A survey of models of network formation: Stability and efficiency. In G. Demange and M. Wooders, editors, Group Formation in Economics: Networks, Clubs, and Coalitions, chapter 1. CUP, 2003.

[14] M. O. Jackson and A. Wolinsky. A strategic model of social and economic networks. Journal of Economic Theory, 71(1):44-74, 1996.

[15] R. Kannan, L. Ray, and S. Sarangi. The structure of information networks. Econ. Theory, 30(1):119-134, 2007.

[16] E. Koutsoupias and C. Papadimitriou. Worst-case equilibria. In Proc. 16th Ann. Symp. on Theoretical Aspects of Comp. Sci. (STAC'99), pages 404-413, 1999.

[17] D. López-Pintado. Network formation, cost-sharing and anti-coordination. International Game Theory Review, 11:53-76, 2009.

[18] M. A. Meléndez-Jiménez. Network formation and coordination: Bargaining and the division of link costs. Technical report, Universidad de Málaga, 2004.

[19] R. B. Myerson. Refinements of the Nash equilibrium concept. Int. J. Game Theory, 7:73-80, 1978.

[20] C. H. Papadimitriou. Algorithms, games, and the Internet. In Proc. 33rd Ann. ACM Symp. on Theory of Comput. (STOC'01), pages 749-753, 2001.

[21] T. Roughgarden. The price of anarchy is independent of the network topology. In Proc. 34th ACM Symp. on the Theory of Computing (STOC'02), pages 428-437, 2002.

[22] O. Tercieux and V. Vannetelbosch. A characterization of stochastically stable networks. Int. J. Game Theory, 34:351-369, 2006. 